\title{
Influence of Calcium Hydroxide Chlorhexidine Combination Vs. Calcium Hydroxide as Intra Ca- nal Medicaments on Postoperative Flare -Up Following two-Visit Endodontic Retreatment Cases: Single Blinded Randomized Clinical Trial
}

\section{Hind Midhat Abdul Gader Hussein*, Sherif Elkhodary and Geraldine Mohamed Ahmed}

Department of Endodontics, Faculty of Dentistry, Cairo University, Cairo, Egypt

*Corresponding Author: Hind Midhat Abdul Gader Hussein, Department of Endodontics, Faculty of Dentistry, Cairo University, Cairo, Egypt.

Received: September 11, 2019; Published: September 30, 2019

\begin{abstract}
Introduction: The aim of the present study was to evaluate the influence of the calcium hydroxide with chlorhexidine combination and calcium hydroxide alone as an intra-canal medicament on postoperative pain and swelling (flare-up) in two visits endodontic retreatment cases.

Methods: Twenty four patients, aged from 20 - 50 years old with no gender prediction, suffering from failed previous endodontic treatment were included in this study. After confirming the diagnosis clinically and radiographically, patients were randomly assigned into two equal groups of 12 patients each. After removal of the previous root canal filling, root canals were prepared and cleaned with $2.5 \%$ sodium hypochlorite, root canals were medicated with calcium hydroxide chlorhexidine combination in the intervention group, whereas the comparator group was medicated with calcium hydroxide as intracanal medicaments for 7 days. Endodontic treatment was completed in the second visits and lateral condensation technique with ADSEAL resin sealer used for obturation. Pain was assessed postoperatively after 6, 12, 24, 48, 72 hours and at 7 days before obturation. Patients were given an analgesic (Ibuprofen $200 \mathrm{mg}$ ) in case of severe pain. Categorical Scale for pain measuring and presence and absence of swelling was used as the primary outcome measure.

Results: Results showed statistically significant difference between the two groups at the first 6 hours with calcium hydroxide Ca group recorded high incidence of postoperative flare up, and no statistically significant difference at 12, 24, 48, 72 , and 7 days between the two groups in categorical scale or swelling records. There was no statistically significant difference in the intake of analgesics between the two groups.

Conclusion: The study concluded that calcium hydroxide chlorhexidine combination resulted in better pain control. Also there was no difference in prevalence of swelling and in the intake of analgesics between the two groups.

Keywords: Endodontic Retreatment; Calcium Hydroxide; Calcium Hydroxide Chlorhexidine; Intra-Canal Medicament; Postoperative Flare-Up
\end{abstract}

\section{Abbreviations}

$\mathrm{Ca}(\mathrm{OH}) 2$ /CHX: Calcium Hydroxide Chlorohexidine Combination; $\mathrm{Ca}(\mathrm{OH}) 2$ : Calcium Hydroxide; CHX: Chlorhexidine; EDTA: Ethylene Diamine Tetra Acetic Acid

\section{Introduction}

Post-operative pain of root canal treated teeth is defined as pain of any degree after initiation of endodontic treatment [1]. Pain after endodontic treatment occurs for several reasons as dur- 
ing intra-appointment or post-obturation in endodontic treatment or as endodontic flare-ups. The endodontic flare ups are defined as strong pain with or without swelling that occurs after initiation or continuation of root canal treatment [2]. Occurrence of post-operative pain can be very unpleasant for both patients and clinicians [3].

The incidence of postoperative pain varies ranging from $1.9 \%$ to $48 \%$ depending on various factors include single visit versus multiple visit endodontic treatment [4]. Other factors include type of intra-canal medicament, root canal instrumentation protocol (enlargement/non enlargement of apical foramen) and status of the pulp [5].

Although postoperative pain associated with root canal therapy is a poor indicator of long-term success, the occurrence and the control of pain are very crucial in endodontics [6]. Certain factors such as preoperative pain, number of appointments, use of intra-canal medications and tooth location may predispose the development of postoperative pain and flare-ups [2].

Flare-ups can occur after root canal treatment and consist of acute exacerbations of an asymptomatic pulpal and/or peri radicular pathologic condition. The causative factors of interappointment pain encompass mechanical, chemical, and/or microbial injury to the pulp or peri radicular tissues. Microorganisms can participate in causation of interappointment pain in the following situations: apical extrusion of debris; incomplete instrumentation leading to changes in the endodontic microbiota or in environmental conditions; and secondary intraarticular infections. Interappointment pain is almost exclusively due to the development of acute inflammation at the peri radicular tissues in response to an increase in the intensity of injury coming from the root canal system [7].

The nonsurgical retreatment for previously failed endodontic therapy provides favourable results [8]. Bacterial infection of the dental pulp may lead to periapical pathosis [9]. Postoperative pain is the result of acute inflammation in the peri radicular tissue caused by the penetration of microorganisms from the root canal during endodontic retreatment [10]. Re-treatment procedure has been suggested as a contributing factor for developing post treat- ment complications. The rate of flare-ups in retreatment cases are reported to be significantly higher than initial root canal treatments [11].

When endodontic treatment fails, nonsurgical endodontic retreatment is often preferred to surgery or extraction [9].

Intra-radicular infection is the most common cause for endodontic failure. It can occur due to insufficient root canal cleaning and persistence of bacteria at the time of root filling or due to reinfection caused by coronal leakage [12].

The most frequent microorganism, isolated in failed cases is $\mathrm{E}$. faecalis, which is a species scarcely found in untreated root canals. It is suggested that E. faecalis is inserted into the canal during root canal treatment. In addition, E. faecalis can withstand intra-canal medication, even calcium hydroxide and luck of symbiotic bacteria. Therefore, it is difficult to be eradicated after its settlement in the canal [12].

The success of endodontic treatment depends on the reduction or elimination of micro-organisms from the root canal system which is achieved by chemo-mechanical preparation [1]. The chemical preparation involves the use of irrigating solutions and intracanal medicaments. Calcium hydroxide is one of the most widely used intra-canal medication in patients undergoing multiple visit endodontic treatment. Its antibacterial action is via release of hydroxyl ions, which kill or inactivate bacteria [13]

Calcium hydroxide is ineffective against all bacterial species especially E. faecalis which is found in the root canal [1]. E. faecalis, have been found to be associated with chronic periodontitis and failed root canal treatments involving chronic apical periodontitis [14]. The prevalence of $E$. faecalis in failed endodontic cases ranged between 24 and $70 \%$ [15]. So it was suggested that calcium hydroxide should be used in combination with other medicaments to enhance its efficacy [11]. The efficacy of calcium hydroxide is affected by its dissociation capability which is determined by the type of vehicle used to mix it [11]. It has been observed that the mixture of calcium hydroxide and chlorhexidine showed synergistic action and greater efficacy than calcium hydroxide alone [1]. 


\section{Subjects and Methods}

Ethics

The protocol of this randomized clinical trial was approved by the institutional review boards/ethical committees (IRBs/ECs) of the Faculty of Dentistry, Cairo University.

\section{Sample size}

The sample size was calculated by the G power program (Universidad Düsseldorf, Düsseldorf, Germany). We will need to study 10 subjects in each group to be able to reject the null hypothesis that the population means of the experimental and control groups are equal with probability (power) 0.8 and type I error probability equal to 0.05 . This number is to be increased to 12 in each group to correct for non-parametric usage; and to 14 to compensate for possible losses during follow up.

\section{Selection of subjects}

All included patients signed an informed consent after the explanation of the involved procedures and the possible risks. Interventions were done by a master's degree student in the Department of Endodontics. Twenty-four participants recruited from the outpatient clinic of the endodontic department, Faculty of Dentistry, Cairo University, in the duration between February 2017 and January 2018.

\section{Inclusion and exclusion criteria}

Inclusion criteria involved patients complaining from signs and symptoms of endodontic treatment failure, patients who had defective root canal filling, patients who were in good health with no systemic disease, patients age range between 20 and 50 years, patients who could understand the categorical tool (points) for measurement in the pain diary and were able to sign the informed consent.

Patient exclusion criteria included those with complicated systemic disease; with severe pain and/or acute apical abscesses; Age under 18 years old; those administered antibiotics or corticosteroids; with multiple teeth that requires retreatment to eliminate the possibility of pain referral and who had root canals that could not be retreated; tooth with broken file or ledges.

Diagnostic criteria of retreatment procedure confirmed by the history of the chief complaint, clinical and radiographic examina- tions. Patients who complained from pain and/or swelling with history of previous treatment or referred from the prosthodontics department for retreatment due to inadequate root canal filling were included in the study according to the eligibility criteria.

\section{Randomization, allocation concealment and blinding}

For randomization, a table of random numbers (from 1 to 24) distributed using computer sequence generation (Microsoft Excel) into group I and group II with participants numbers (12 numbers in each group). The table was kept with the assistant supervisor. The patient was blinded (single-blind study). After the subject was confirmed to be enrolled in the trial, the investigator gave a phone call to the co-supervisor who allocated the patient either to the intervention or the control group according to the generated random sequence. The investigator was the one responsible for performing the whole procedure, assessing the outcomes from the patients and recording any abnormal findings such as mishaps or side effects.

\section{Operating sequence}

\section{First session}

The tooth was properly isolated using rubber dam. The previous restorations were removed using high-speed hand piece and sterilized round bur under coolant. Modification of the access cavity was performed using Endo-Z bur. Removal of the filling material in the root canal was performed as follows: Few drops of the gutta-percha Carven solvent were injected at the canal entrance by disposable syringe with gauge 27 open needle and left for 2 minutes to allow for penetration. Then Protaper Universal System F3 and F2 files were adjusted in an NSK endo motor with the speed adjusted at $300 \mathrm{rpm}$ with torque 2 adjusted according to the manufacturer's instructions. Used in the coronal 2/3 of the canal in an up and down motion, sequentially. All the files introduced to the point where resistance felt by the filling materials after which the file removed, cleaned and reinserted with addition of solvent. Hedstrom hand files sizes 40, 35 and 30 were then used to complete the removal of the apical part of the canal in a crown down technique. Irrigation with $3 \mathrm{ml} 2.5 \% \mathrm{Na}$ OCl with each $1 \mathrm{ml}$ in $60 \mathrm{sec}$ using gauge 27 side vented needle between successive files. It was prepared by adding $10 \mathrm{ml}$ of sterile distilled water to $10 \mathrm{ml}$ of $5.25 \%$ sodium hypochlorite solution. After removal of the filling material, the working lengths were determined by apex locater and confirmed by periapical radiograph $1 \mathrm{~mm}$ short from the radiograph apex. Chemo-me- 
chanical preparation using Protaper Universal rotary system. The finishing files were F1 (tip size 20), F2 (tip size 25) and F3 (tip size 30 ) were used in a pecking motion canals expect the mesial canals in molars where the finishing file was F2. Preparation of the canal was considered complete using ISO files with master apical file corresponding to size \#40 in palatal maxillary molars and distal canals in the mandibular molars and \#35 in the remaining canals of molar. Irrigation with $3 \mathrm{ml} 2.5 \% \mathrm{Na} \mathrm{OCl}$ with each $1 \mathrm{ml}$ per $60 \mathrm{sec}$ using gauge 27 side vented needle between successive files. Then the final irrigation was made by saline and root canals were dried with Protaper special paper points. Then, cases were classified to 2 groups according to medicament used into:

\section{Group (a): control group}

A Metapex calcium hydroxide injectable (calcium hydroxide with iodoform) was used. The canals were dried with paper points. The paste directly poured in a Met biomed syringe with disposable tip was injected in the root canal system. A lentulo spiral connected to low speed headpiece was used with sizes 30 or 35 corresponding to the master apical file, then introduced into the root canal and slowly rotated in a clock wise motion into the canal $2 \mathrm{~mm}$ short of the working length. The procedure was repeated until the paste was observed at the canal orifice.

\section{Group (b): In the experimental group}

Calcium hydroxide chlorohexidine combination $\left(\mathrm{Ca}(\mathrm{OH})_{2} /\right.$ CHX) was used. The canals were dried with paper points. A paste consists of $1.2 \mathrm{~g}$ powder of $\mathrm{Ca}(\mathrm{OH})_{2}$ mixed GLUO-CHX ${ }_{2} \%$ chlorohexidine gel mixed in 1:1 ratio on a paper pad and then plugged in to the canal by using a lentulo spiral connected to low speed headpiece was used with sizes 30 or 35 corresponding to the master apical file, then introduced into the root canal and slowly rotated in a clock wise motion into the canal $2 \mathrm{~mm}$ short of the working length. The procedure was repeated until the paste was observed at the canal orifice. Excess medication was removed from the pulp chamber with a sterile cotton pellet. A sterile cotton pellet placed over the pulp chamber and a Den Seal glass ionomer was used to seal the cavity. No systemic medication was prescribed, the patient were instructed to take mild analgesics if they experienced unbearable pain (severe pain) ibuprofen $(200 \mathrm{mg}$ ) but after contacting the operator. One week after initial appointment the patients were recalled to return for completion of endodontic treatment. The pain recorded after $6,12,24,48,72$ hours through a telephone call and at
7 days pre obturation as mentioned in the pain dairy above. Patient with sever postoperative pain and/or occurrence of swelling were classified as flare-up [16].

\section{Second session}

Rubber dam was applied for thorough isolation of the root canal system. Glass ionomer and the cotton pellet were removed from the pulp chamber. Removal of the intra-canal medicament was performed using a hand K-file corresponding to the size of master apical file and irrigation with $3 \mathrm{ml} 2.5 \%$ sodium hypochlorite solution between every subsequent instrument. Then, canals flushed with normal saline $3 \mathrm{ml}$. After that, each root was rinsed with 17\% EDTA solution $1 \mathrm{ml}$ for 3 minute then followed by saline as a final rinse. Master cone corresponding to the master apical file determined and confirmed using periapical X-ray. Canals were then dried with sterile paper points and obturation performed using gutta-percha points and $\mathrm{AD}$ seal sealer with lateral compaction technique. A spreader of suitable size was selected and used to allow space for auxiliary cones, together with a resin-based root canal sealer. The access cavity was sealed using sterile dry cotton on the floor and the glass ionomer as a temporary restoration. Postoperative radiograph was taken to confirm the density and length of the filling material. Patients were instructed to complete the treatment procedures by placing a permanent restoration followed by a fullcoverage restoration in fixed prosthodontic clinic of Faculty of Dentistry, Cairo University.

Pain assessment and outcomes

Postoperative pain

Subjectively measured using a categorical scale of 4 classes:

1. No pain;

2. Mild pain: recognizable but not discomforting pain that required no analgesics;

3. Moderate pain: discomforting but bearable pain (analgesics if used were effective in relieving pain);

4. Severe pain: difficult to bear (analgesics were effective in relieving pain) [3].

\section{Postoperative swelling}

Categorized as presence or absence of swelling will be measured after $1^{\text {st }}$ visit at $6 \mathrm{~h}, 12 \mathrm{~h}, 24 \mathrm{~h}, 48 \mathrm{~h}$, and $72 \mathrm{~h}$ and after 7 days from $1^{\text {st }}$ visit: Pre-obturation. 
Influence of Calcium Hydroxide Chlorhexidine Combination Vs. Calcium Hydroxide as Intra Canal Medicaments on Postoperative Flare -Up Following two-Visit Endodontic Retreatment Cases: Single Blinded Randomized Clinical Trial

\section{Objectively}

Percussion test (presence or absence of pain).

Primary outcomes

Postoperative flare-up (pain or/and swelling) will be measured using the categorical scale $\mathrm{s}$ as mentioned above after $6 \mathrm{hrs}, 12 \mathrm{hrs}$., $24 \mathrm{hr}$., 48hr, $72 \mathrm{hr}$., and 7 days pre obturation. With severe pain and/or swelling considered as flare-up.

\section{Secondary outcomes}

Recording analgesic intake as well as number of analgesic tables.

\section{Statistical analysis}

Qualitative data were presented as frequencies and percentages. Fisher's Exact test was used for comparisons between the two groups. Friedman's test was used to study the changes by time in each group. Numerical data were explored for normality by checking the distribution of data and using tests of normality (Kolmogorov-Smirnov and Shapiro-Wilk tests). Age data showed normal (parametric) distribution. Parametric data were presented as mean and standard deviation (SD) values and compared using Student's t-test.

The significance level was set at $\mathrm{P} \leq 0.05$. Statistical analysis was performed with IBM $^{\circledR}$ SPSS $^{\circledR}$ Statistics Version 20 for Windows.

\section{Results}

The trial design followed the CONSORT 2010. The flow of the participants throughout the study is presented in (Figure 1).

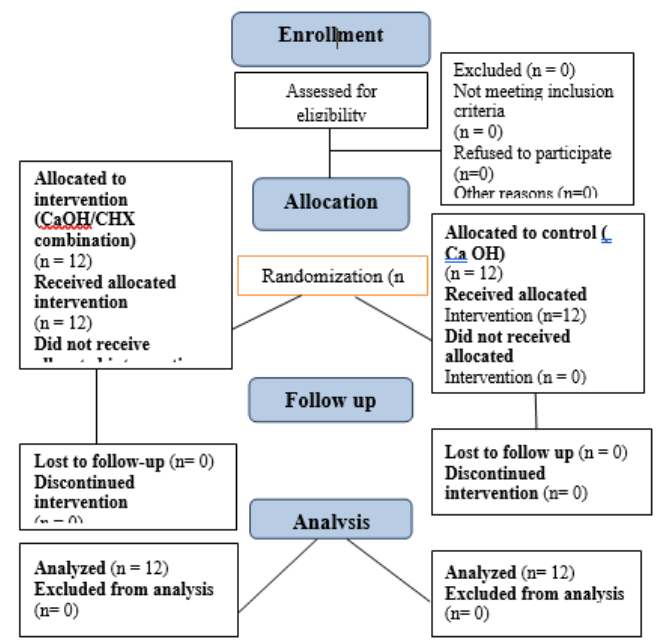

Figure 1: Consort 2010 flow diagram of the trial design.
Postoperative pain

1. Demographic data (age, gender and tooth type distribution) illustrated in table 1.

2. There was no statistically significant difference regarding mean age values (P-value $=0.414$ ) as well as gender distribution $(\mathrm{P}$-value $=1.000)$ between the two group.

3. Severity of pain.

\begin{tabular}{|c|c|c|c|}
\hline & $\begin{array}{l}\mathrm{Ca}(\mathrm{OH})_{2} \\
(\mathrm{n}=12)\end{array}$ & $\begin{array}{l}\mathrm{Ca}(\mathrm{OH})_{2}+\mathrm{CHX} \\
(\mathrm{n}=12)\end{array}$ & $P$-value \\
\hline \multicolumn{4}{|l|}{ Age (Years) } \\
\hline Mean (SD) & $36.5(5.9)$ & $34.3(7.2)$ & 0.414 \\
\hline Gender [n (\%)] & & & \multirow{3}{*}{1.000} \\
\hline Male & $4(33.3 \%)$ & $4(33.3 \%)$ & \\
\hline Female & $8(66.7 \%)$ & $8(66.7 \%)$ & \\
\hline
\end{tabular}

Table 1: Mean, standard deviation (SD), frequencies (n), percentages and results of Student's t-test and Fisher's exact tests for comparisons of demographic data between the two groups.

$*$ : Significant at $\mathrm{P} \leq 0.05$.

Comparison between the two groups

- $\quad$ After 6 hours, there was a statistically significant difference between the two groups (P-value $=0.046)$. Ca $(\mathrm{OH}) 2$ group showed higher prevalence of severe pain than $\mathrm{Ca}(\mathrm{OH}) 2+\mathrm{CHX}$ group.

- $\quad$ After 12 hours, there was no statistically significant difference between the two groups (P-value $=1.000)$.

- $\quad$ After 24 hours, there was no statistically significant difference between the two groups (P-value $=1.000)$.

- After 48 hours, there was no statistically significant difference between the two groups (P-value $=1.000)$.

- $\quad$ After 72 hours, there was no statistically significant difference between the two groups (P-value $=1.000)$.

- $\quad$ At day 7, all cases showed no pain. Hence, no statistical comparison was performed.

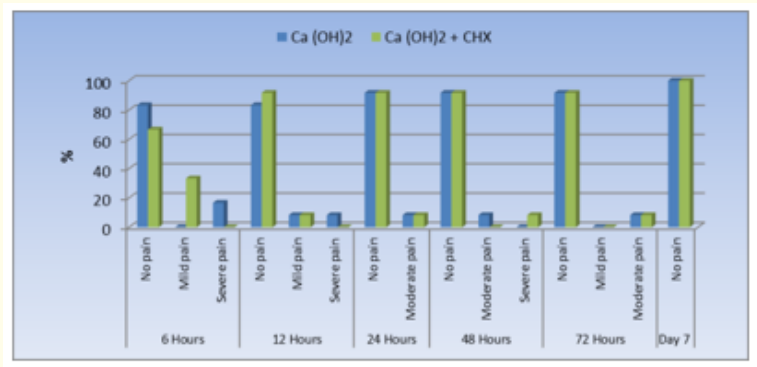

Figure 2: Bar chart representing severity of pain in the two groups. 


\section{Changes by time in $\mathrm{Ca}(\mathrm{OH})_{2}$ group}

There was no statistically significant change in severity of pain by time in $\mathrm{Ca}(\mathrm{OH})_{2}$ group (P-value $\left.=0.104\right)$.

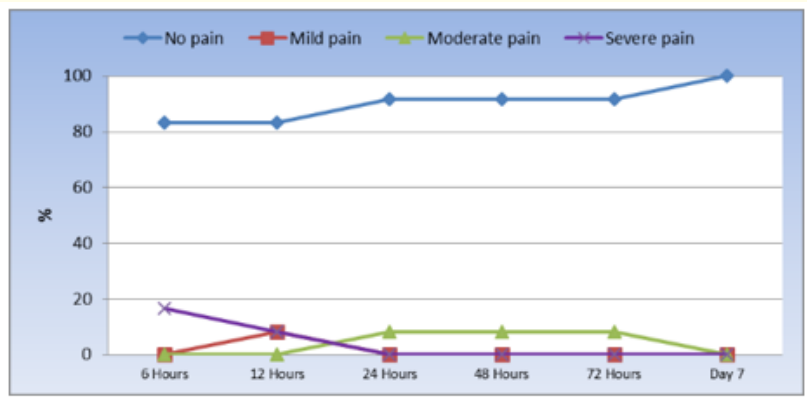

Figure 3: Line chart representing change by time in severity of pain within $\mathrm{Ca}(\mathrm{OH})_{2}$ group.

\section{Changes by time in $\mathrm{Ca}(\mathrm{OH})_{2}+\mathrm{CHX}$ group}

There was no statistically significant change in severity of pain by time in $\mathrm{Ca}(\mathrm{OH})_{2}+\mathrm{CHX}$ group $(\mathrm{P}$-value $=0.133)$.

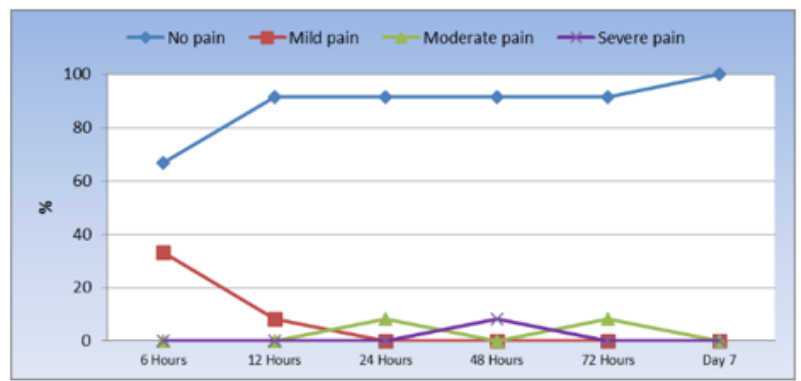

Figure 4: Line chart representing changes by time in severity of pain within $\mathrm{Ca}(\mathrm{OH})_{2}+\mathrm{CHX}$ group.

\section{Swelling}

There was no statistically significant difference between prevalence of swelling in the two groups $(\mathrm{P}$-value $=1.000)$ (Figure 5).

\section{Intake of analgesics}

There was no statistically significant difference between intake of analgesics in the two groups (P-value $=1.000$ ) (Figure 6).

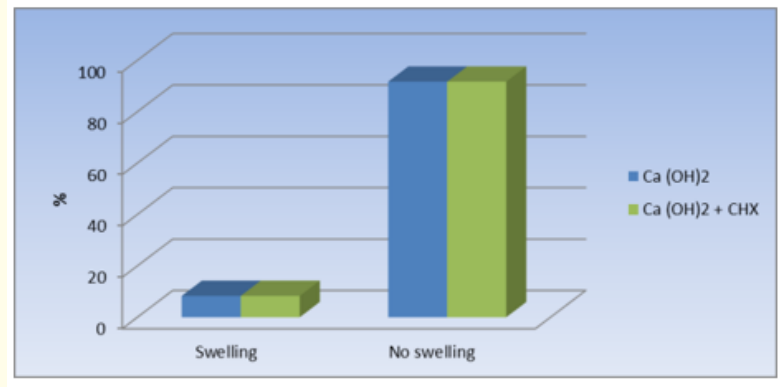

Figure 5: Bar chart representing prevalence of swelling in the two groups.

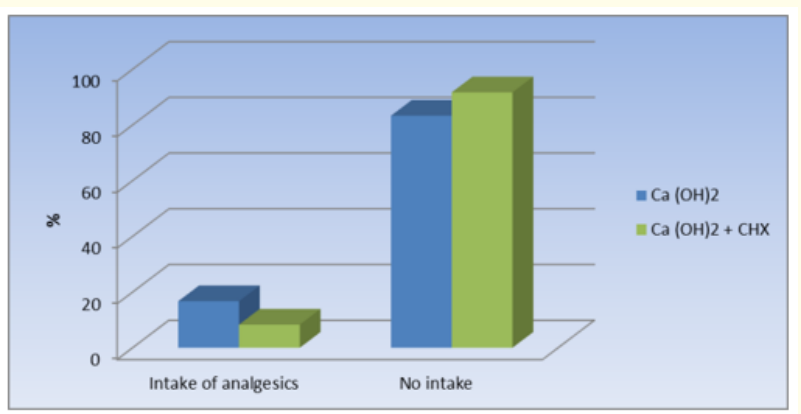

Figure 6: Bar chart representing intake of analgesics in the two groups.

\section{Discussion}

Teeth with poor endodontic treatment contain a great number of bacterial species [17] especially E. faecalis which has been detected in $70 \%$ in failed endodontic cases. The aim of the present study was to evaluate the influence of the $\mathrm{Ca}(\mathrm{OH})_{2} / \mathrm{CHX}$ combination and $\mathrm{Ca}(\mathrm{OH})_{2}$ as an intra-canal medicament on postoperative pain and swelling (flare-up) in two visits endodontic retreatment cases.

In our study intra-canal medicaments were used between visits to deny space for microbial proliferation between visits and can play an important role in preventing the recontamination of the root canals between appointments. And prevent postoperative pain caused by persistent intracanal micro-organisms or by secondary microbial invaders [17]. 
Considering age as one of the significant effects on the postoperative pain, patients aging between 20 and 50 years old were selected [18]. Patients has undergone their retreatment procedure within two visits, targeting to gain antibacterial effect by using intracanal medicament, reduce postoperative pain, and diminish the number of flare-ups [17].

Various methods have been used for the removal of root canal filling materials, which are broadly classified into thermal, mechanical, chemical and a combination of the three. In this study, a combination of chemical (Carvene solvent) and mechanical (Protaper universal rotary system (PTU) and Hedstorm files) were used. In which, solvents can soften and dissolve gutta-percha present in the root canal to facilitate its removal and allow for the penetration of manual and rotary instruments [19].

Pro Taper rotary files have proven their efficiency in removing gutta-percha because of the association of the solvent with the instrument rotation, which causes the dilution and plasticization of gutta-percha [20]. Therefore Pro Taper Universal files were used since they were faster than the Pro Taper retreatment files to perform GP removal in the retreatment of teeth obturated according to the single master cone technique, and both techniques promoted similar apical extrusion of debris [21].

In the present study, the working length was determined by IPEX electronic apex locator, due to its reliability, and then confirmed by the radiograph [22]. Root canal were irrigated using $2.5 \%$ sodium hypochlorite $(\mathrm{Na} \mathrm{OCl})$ since it's capable to reduce the intracanal microbiota [23]. Side vented needle was used to lower the incidence of irritant extrusion in to the periapical space [24] The smear layer was removed by rinsing the canal with $1 \mathrm{~mL} \mathrm{17 \%}$ EDTA and then leaving the canal filled with this solution for 3 minutes [25].

Despite the fact that $\mathrm{Ca}(\mathrm{OH})_{2}$ is one of the most widely used an intracanal medicament, due to its high $\mathrm{pH}$ and potent antibacterial activity [26], however it was reported to be ineffective against all bacterial species especially E. faecalis found in the root canal [1]. Therefore it was demonstrated that the mixture of $\mathrm{Ca}(\mathrm{OH})_{2} / \mathrm{CHX}$ combination showed a synergistic action and greater efficacy than calcium hydroxide alone [27]. Lentulo spirals used for placement of intra-canal medicament resulted in less voids (28) and better packing of paste [29]. Pain assessment was performed using categorical scale of 4 classes as a modification to the visual analogy scale (VAS) for its reliability, validity and simplicity in pain rating [17].

The result of this study showed that after 6 hours, there was a statistically significant difference between the $\mathrm{Ca}(\mathrm{OH})_{2} / \mathrm{CHX}$ combination group and $\mathrm{Ca}(\mathrm{OH})_{2}$ group. Where the CA OH group showed higher prevalence of severe pain than $\mathrm{Ca}(\mathrm{OH})_{2} / \mathrm{CHX}$ combination group. Our findings in agreement with [30] who reported that a combination of $\mathrm{Ca}(\mathrm{OH})_{2}$ and $2 \%$ CHX was significantly different in reducing inter-appointment pain than that of $\mathrm{Ca}(\mathrm{OH})_{2}$ and saline group. It might be due to its high $\mathrm{pH}$, suggesting an increase of the ionized capacity of the CHX molecule. Moreover, the addition of CHX to $\mathrm{Ca}(\mathrm{OH})_{2}$ lowers its contact angle and improves the wettability of the medication on the root canal. This could also be because of synergistic effect of $\mathrm{Ca}(\mathrm{OH})_{2}$ and $\mathrm{CHX}$ on liposaccharides/ endotoxins produced by gram negative bacteria. Hence, both the intracanal medications complement their actions [31].

Our results showed no statistically significant difference after $12,24,48,72$ hours between $\mathrm{Ca}(\mathrm{OH})_{2} / \mathrm{CHX}$ combination group and $\mathrm{Ca}(\mathrm{OH})_{2}$ group. At day 7 all cases in both groups showed no pain.

In contrast [32] reported that 2\% CHX gel alone is more effective at 72 hours than $\mathrm{Ca}(\mathrm{OH})_{2}$ paste alone or their combination against both organisms. In failed root canal treatments, $2 \% \mathrm{CHX}$ gel may be a more effective intracanal medicament than $\mathrm{Ca}(\mathrm{OH})_{2}$ paste or their combination. This might be due to the difference in $\mathrm{pH}$ of chlorhexidine and calcium hydroxide and also due to the binding of chlorhexidine molecule to calcium hydroxide ions and hence inhibiting the free release of chlorhexidine molecule. In our study, analgesics were only prescribed on demand and not a regular prescription of medication since it would influence the outcome measures of the study $[1,17]$. Among the non-steroidal anti-inflammatory drugs, Ibuprofen was selected because it is effective for treating acute pain and inflammation related to endodontic treatment, rapidly absorbed and metabolized by the liver [33].

\section{Conclusion}

The conclusion of this study is as follows:

- $\quad$ Calcium hydroxide chlorhexidine combination resulted in better pain control.

Citation: Hind Midhat Abdul Gader Hussein., et al. "Influence of Calcium Hydroxide Chlorhexidine Combination Vs. Calcium Hydroxide as Intra Canal Medicaments on Postoperative Flare -Up Following two-Visit Endodontic Retreatment Cases: Single Blinded Randomized Clinical Trial". Acta Scientific Dental Sciences 3.10 (2019): 117-125. 
- $\quad$ There was no difference between prevalence of swelling in the two groups.

- There was no difference in the intake of analgesics between the two groups.

\section{Conflict of Interest}

The authors deny any conflicts of interest in this study.

\section{Bibliography}

1. Menakaya I., et al. "Incidence of postoperative pain after use of calcium hydroxide mixed with normal saline or $0.2 \%$ chlorhexidinedigluconate as intracanal medicament in the treatment of apical periodontitis". Saudi Dental Journal 27.4 (2015): 187-193.

2. Tsesis I., et al. "Flare-ups after endodontic treatment: A metaanalysis of literature". Journal of Endodontics 34.10 (2008): 1177-1181.

3. Mathew S., et al. "Post operative pain in endodontics: A systemic review". Journal of Dentistry and Oral Hygiene 7.8 (2015): 130-137.

4. Ali S., et al. "Prevalence of and factors affecting post obturation pain following single visit root canal treatment in Indian population: A prospective, randomized clinical trial". Contemporary Clinical Dentistry 3.4 (2012): 259-263.

5. Silva E., et al. "Postoperative pain after foraminal enlargement in anterior teeth with necrosis and apical periodontitis: a prospective and randomized clinical trial". Journal of Endodontics 39.2 (2013): 173-176.

6. Yousaf 0., et al. "Post operative pain comparison in single versus two visit endodontic treatment". Pakistan Oral and Dental Journal 36.3 (2016): 272-275.

7. Sipavičiūtė E., et al. "Pain and flare-up after endodontic treatment procedures". Baltic Dental and Maxillofacial Journal 16.1 (2014): 25-30.

8. Moazami F., et al. "Success Rate of Nonsurgical Endodontic Treatment of Nonvital Teeth with Variable Periradicular Lesions". Iranian Endodontic Journal 6.3 (2011): 119-124.

9. Caliskan MK., et al. "Nonsurgical retreatment of teeth with periapical lesions previously managed by either endodontic or surgical intervention". Oral Surg, Oral Med, Oral Pathol, Oral Radiol Endod 100.2 (2015): 242-248.
10. HepsenogluYE., et al. "Postoperative Pain Intensity after Single- versus 2-visit Nonsurgical Endodontic Retreatment: A Randomized Clinical Trial". Journal of Endodontics 44.9 (2018): 1-8.

11. Onay EO., et al. "The evaluation of endodontic flare-ups and their relationship to various risk factors". BMC Oral Health 15 (2015): 142 .

12. Stom BJ., et al. "Non-Surgical Endodontic Retreatment after Unsuccessful Apicectomy: A Case Report". Balkan Journal of Stomatology 15 (2011): $166-170$.

13. Mohammadi Z., et al. "The properties and applications of chlorhexidine in endodontics". Journal of Endodontics 42.2 (2009): 288-302.

14. Wang QQ., et al. "Prevalence of Enterococcus faecalis in saliva and filled root canals of teeth associated with apical periodontitis". International Journal of Oral Science 4.1 (2004): 19-23.

15. Vidana R., et al. "Enterococcus faecalis infection in root canals - host-derived or exogenous source? Letters in Applied Microbiology". The Society for Applied Microbiology (2010).

16. Gama TG., et al. "Postoperative pain following the use of two different intracanal medications". Clinical Oral Investigations 12.4 (2008): 325-330.

17. Yoldas 0., et al. "Postoperative pain after endodontic retreatment: Single-versus two-visit treatment". Oral Surg, Oral Med, Oral Pathol Oral Radiol Endod 98.4 (2004): 483-487.

18. Sipavičiūtė E., et al. "Pain and flare-up after endodontic treatment procedures". Stomatologija, Baltic Dental and Maxillofacial Journal 16.1 (2014): 25-30.

19. Rehman K., et al. "Comparison of orange oil and chloroform as Gutta-percha solvents in endodontc retreatment". The Journal of Contemporary Dental Practice 14.3 (2013): 478-482.

20. Medeiros BJA., et al. "Evaluation of four gutta-percha removal techniques for endodontic retreatment". RSBO Revista Sul Brasileira de Odontologia 11.4 (2014): 340-345.

21. Heling I., et al. "The antimicrobial effect within dentinal tubules of four root canal sealers". Journal of Endodontics 22.5 (1996): 257-259. 
22. Raghu K., et al. "In vivo evaluation of the accuracy of working length determination using an electronic apex locater IPEX (NSK) on vital uninfected teeth and teeth with radiographic evidence of periapical lesions". Journal of International Society of Preventive and Community Dentistry 4.6 (2018): S204- S208.

23. Gomes-Filho JE., et al. "Comparison of the biocompatibility of different root canal irrigants". Journal of Applied Oral Science 16.2 (2008): $137-414$.

24. Yeter KY., et al. "Weight of apically extruded debris following use of two canal instrumentation techniques and two designs of irrigation needles". International Endodontic Journal 46.9 (2013): 795-799.

25. Baca P., et al. "Antimicrobial Substantivity over Time of Chlorhexidine and Cetrimide". Journal of Endodontics 38.7 (2012): 927-930.

26. Fava LRG., et al. "Calcium hydroxide pastes: classification and clinical indications". Journal of Endodontics 32.4 (1999): 257282.

27. Rao N., "Efficacy of calcium hydroxide-chlorhexidene paste against Enterococcus faecalis - An in vitro study". Endodontology 16 (2004): 61-64.

28. Peters CI., et al. "Calcium hydroxide dressings using different preparation and application modes: density and dissolution by simulated tissue pressure". Journal of Endodontics 38.12 (2005): 889-895.

29. Staehle HJ., et al. "Comparative in vitro investigation of different methods for temporary root canal filling with aqueous suspensions of calcium hydroxide". Endodontics and Dental Traumatology 13 (1997): 106-112.

30. Khattak YK., et al. "Comparison of interappointment pain between calcium hydroxide mixed with $2 \%$ chlorhexidine and calcium hydroxide mixed with normal saline; a Randomized controlled trial". Journal of Khyber College of Dentistry 5.1 (2014): 33-37.

31. Maroli S., et al. "An in vivo comparative pain evaluation on using Azadirachtaindica as an intracanal medicament". International Journal of Scientific Research 6.4 (2017): 253-255.
32. Walton RE., et al. "Calcium Hydroxide as an Intracanal Medication: Effect on Posttreatment Pain”. Journal of Endodontics 29 (2003): 627-629.

33. Moore ND., et al. "In search of an ideal analgesic for common acute pain”. Acute Pain 11.3-4 (2009): 129-137.

\section{Volume 3 Issue 10 October 2019}

(C) All rights are reserved by Hind Midhat Abdul Gader Hussein., et al. 